\title{
Lipoprotein(a) levels over time A long-term follow-up study of a large cohort of children
}

\author{
Lotte M. de Boer ${ }^{1,2}$, Michel H. Hof ${ }^{1}$, Albert Wiegman², John J. Kastelein ${ }^{3}$, Barbara A. Hutten ${ }^{1}$ \\ Departments of Clinical Epidemiology and Biostatistics ${ }^{1}$, Pediatrics ${ }^{2}$ and Vascular medicine ${ }^{3}$ \\ Academic Medical Center, Amsterdam
}

\section{INTRODUCTION}

- The European Atherosclerosis Society (EAS) recommends measuring Lp(a) levels in patients with intermediate or high cardiovascular risk. In clinical practice $\operatorname{Lp}(\mathrm{a})$ is often measured once in these patients, assuming that it is stable over time. However, several studies reported within-person fluctuations of $L p(a)$ levels, showing that $L p(a)$ does not remain constant over time.

- Therefore, we evaluated $L p(a)$ levels in a large cohort of children who were followed-up into adulthood if $L p(a)$ levels remain constant with increasing age and if $L p(a)$ levels fluctuate within the individual patient.

AIM: to evaluate whether $\operatorname{Lp}(a)$ levels remain constant with increasing age and if $L p(a)$ levels fluctuate within the individual patient

\section{METHODS}

- Study population and design: we performed a retrospective cohort study in children that were referred to the outpatient clinic of the Academic Medical Center for a tentative diagnosis of inherited dyslipidemia between 1989 and 2017.

- Statistics: Linear mixed models were used to evaluate $L p(a)$ levels with increasing age and intra-individual changes in $\mathrm{Lp}(\mathrm{a})$ levels over time.

\section{RESULTS}

Table 1. Demographic and clinical characteristics at first visit

\begin{tabular}{ll}
\hline & $\begin{array}{l}\text { Study population } \\
\mathbf{n}=\mathbf{2 , 8 1 3}\end{array}$ \\
\hline Male gender - no (\%) & $1,424(51)$ \\
Mean age (years) - mean (SD) & $10.1(3.6)$ \\
Body mass index (kg/m2) - mean (SD) & $18.3(3.9)$ \\
Systolic blood pressure (mmHg) - mean (SD) & $110(12)$ \\
Diastolic blood pressure (mmHg) - mean (SD) & $65(9)$ \\
Lipids and lipoproteins & \\
Total cholesterol (mmol/L) - mean (SD) & $6.4(1.9)$ \\
LDL-cholesterol (mmol/L) - mean (SD) & $4.7(1.9)$ \\
HDL -cholesterol (mmol/L) - mean (SD) & $1.4(0.3)$ \\
Triglycerides (mmol/L) - median (IQR) & $0.66(0.46-0.97)$ \\
Lipoprotein (a)* (mg/L) - median (IQR) & $143(62-380)$ \\
Diagnosis - no (\%) & \\
Molecular proven non-FH & $285(10)$ \\
Probably non-FH & $363(13)$ \\
Highly probable FH & $136(5)$ \\
Molecular proven heterozygous FH & $1,923(69)$ \\
Molecular proven homozygous FH & $18(1)$ \\
Other causes of dyslipidemia & $65(2)$ \\
Statins - no (\%) & $576(21)$ \\
\hline interquartile range: FH: familial hypercholesterolemia *At first measurement
\end{tabular}

Table 2. Percental and absolute change in $L p(a)$ levels for a child of 10 years at the age of $11,15,20$ and 30 years $(n=2,813)$

\begin{tabular}{llll}
\hline & \% Change* & Mean Lp(a) levels* & $\begin{array}{l}\text { Mean Lp(a) levels } \\
\text { (example) }\end{array}$ \\
\hline Child of 10 years (reference) & - & $149 \mathrm{mg} / \mathrm{L}$ & $470 \mathrm{mg} / \mathrm{L}$ \\
At age 11 & $1.2(0.8-1.5)$ & $151 \mathrm{mg} / \mathrm{L}$ & $476 \mathrm{mg} / \mathrm{L}$ \\
At age 15 & $5.9(4.2-7.7)$ & $158 \mathrm{mg} / \mathrm{L}$ & $498 \mathrm{mg} / \mathrm{L}$ \\
At age 20 & $12.2(8.5-16.0)$ & $167 \mathrm{mg} / \mathrm{L}$ & $527 \mathrm{mg} / \mathrm{L}$ \\
At age 30 & $25.9(17.7-34.6)$ & $188 \mathrm{mg} / \mathrm{L}$ & $592 \mathrm{mg} / \mathrm{L}$ \\
\hline
\end{tabular}

*Estimated from the model

Figure 1. The regression line (95\% confidence interval) when $L p(a)$ measurements are plotted against age

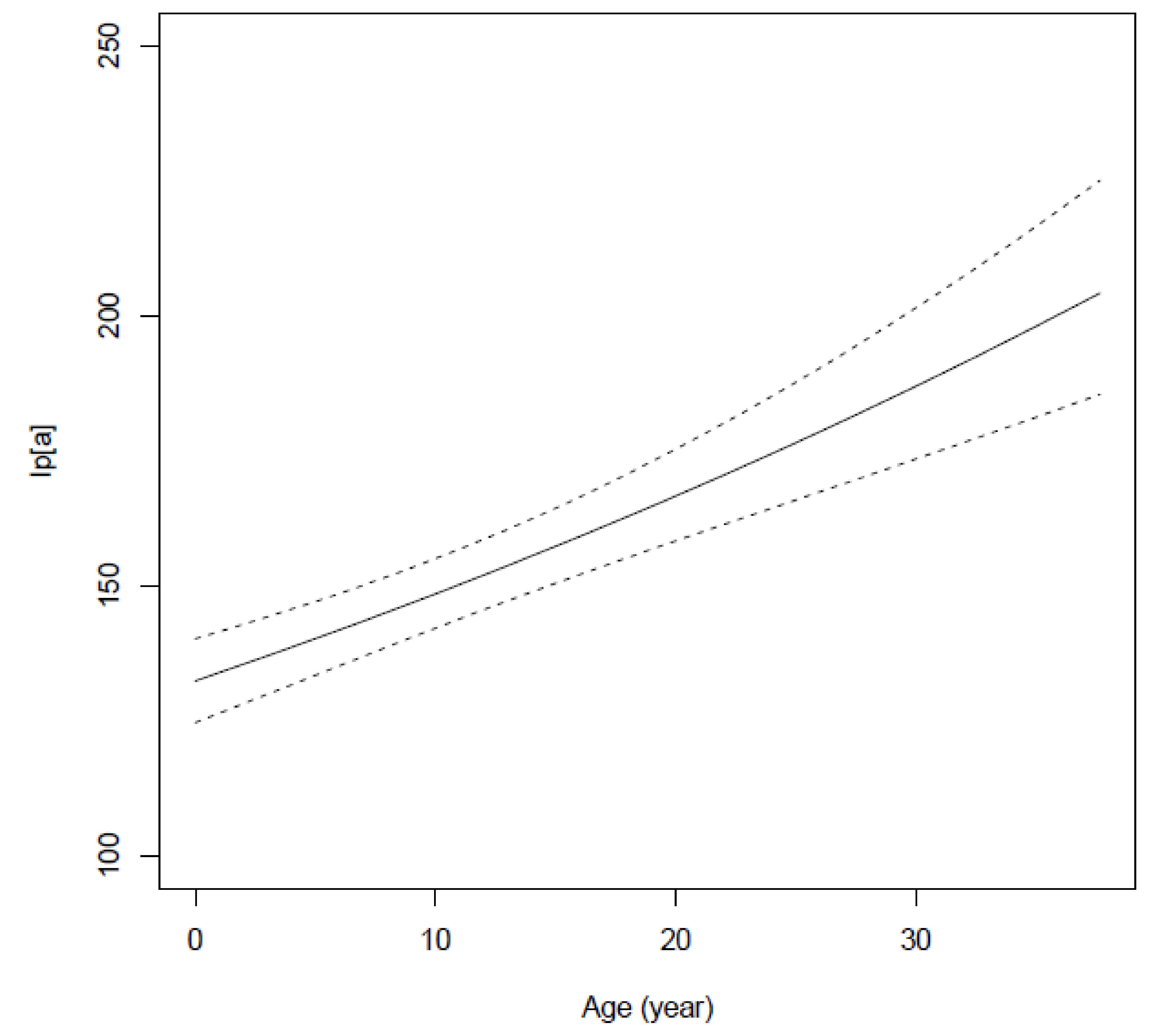

Mixed models analysis showed within-subject variation of $37.4 \%$ (95\% Cl: 36.2 - 38.7)

\section{CONCLUSION}

